\title{
Rorc restrains the potency of ST2+ regulatory $T$ cells in ameliorating intestinal graft-versus-host disease
}

\author{
Jinfeng Yang, ${ }^{1}$ Abdulraouf Ramadan, ${ }^{1}$ Dawn K. Reichenbach, ${ }^{2}$ Michael Loschi, ${ }^{2}$ jilu Zhang, ${ }^{1}$ \\ Brad Griesenauer, ${ }^{1}$ Hong Liu, ${ }^{1}$ Keli L. Hippen, ${ }^{2}$ Bruce R Blazar, ${ }^{2}$ and Sophie Paczesny' \\ 'Department of Pediatrics, Indiana University School of Medicine, Indianapolis, Indiana, USA. ${ }^{2}$ Department of Pediatrics, \\ University of Minnesota, Minneapolis, Minnesota, USA.
}

\begin{abstract}
Soluble stimulation-2 (ST2) is increased during graft-versus-host disease (GVHD), while Tregs that express ST2 prevent GVHD through unknown mechanisms. Transplantation of Foxp ${ }^{-} \mathrm{T}$ cells and Tregs that were collected and sorted from different Foxp3 reporter mice indicated that in mice that developed GVHD, ST2 ${ }^{+}$Tregs were thymus derived and predominantly localized to the intestine. ST2 $2^{-1-}$ Treg transplantation was associated with reduced total intestinal Treg frequency and activation. ST2 $2^{--}$versus WT intestinal Treg transcriptomes showed decreased Treg functional markers and, reciprocally, increased Rorc expression. Rorc ${ }^{-1-} \mathrm{T}$ cells transplantation enhanced the frequency and function of intestinal ST2 ${ }^{+}$Tregs and reduced GVHD through decreased gutinfiltrating soluble ST2-producing type 1 and increased IL-4/IL-10-producing type $2 \mathrm{~T}$ cells. Cotransfer of ST2 $2^{+}$Tregs sorted from Rorc ${ }^{-1-}$ mice with WT CD25-depleted T cells decreased GVHD severity and mortality, increased intestinal ST2 ${ }^{+} K L R G 1^{+}$Tregs, and decreased type $1 \mathrm{~T}$ cells after transplantation, indicating an intrinsic mechanism. Ex vivo IL-33-stimulated Tregs (Treg ${ }_{\mid-33}$ ) expressed higher amphiregulin and displayed better immunosuppression, and adoptive transfer prevented GVHD better than control Tregs or Treg ${ }_{\mathrm{IL}-33}$ cultured with IL-23/IL-17. Amphiregulin blockade by neutralizing antibody in vivo abolished the protective effect of Treg $_{\mid-33^{3}}$. Our data show that inverse expression of ST2 and RORyt in intestinal Tregs determines $\mathrm{CVHD}$ and that Treg $\mathrm{IL-33}_{3}$ has potential as a cellular therapy avenue for preventing GVHD.
\end{abstract}

Authorship note: JY, AR, DKR, and ML contributed equally to this work. BRB and SP contributed equally to this work.

Conflict of interest: SP has a patent (US20130115232A1), "Methods of detection of graft-versus-host disease," licensed to Viracor-IBT Laboratories.

License: Copyright 2019, American Society for Clinical Investigation.

Submitted: May 3, 2018 Accepted: January 25, 2019 Published: March 7, 2019

Reference information: JCI Insight. 2019;4(5):e122014. https://doi.org/10.1172/jci. insight.122014.

\section{Introduction}

Allogeneic hematopoietic cell transplantation (allo-HCT) is an essential therapeutic modality for patients with hematological malignancies and benign blood disorders (1). Unfortunately, graft-versus-host disease (GVHD) remains the major complication of allo-HCT and is associated with high mortality, morbidity, and health care costs $(2,3)$. Several studies have demonstrated that $\mathrm{CD} 4^{+} \mathrm{Foxp} 3^{+}$Tregs inhibit proliferation of conventional T cells (Tcons), limiting acute GVHD (aGVHD) lethality while preserving graph-versus-leukemia (GVL) activity (4-7). Clinical adoptive transfer experiments showed the safety and efficacy of Treg infusions (8-10), suggesting that Treg-based cellular therapy is a promising approach for aGVHD prevention. Despite research into the role of Tregs in aGVHD, the precise mechanisms through which Tregs control alloreactive immune responses have not been fully elucidated. Tregs are heterogeneous populations that show a high degree of phenotypic and functional diversity (11). Two studies have shown that transplantation of CD62 $\mathrm{L}^{\text {hi }}$ Tregs into irradiated MHC-mismatched recipients protected them from lethal aGVHD because the Tregs interfered with the activation and expansion of effector T cells (Teffs) in secondary lymphoid organs early after HCT $(12,13)$. However, the functional nonlymphoid tissue Treg subsets that have the greatest impact on intestinal aGVHD remain largely unknown. Based on the location of induction, Tregs can be divided into two different subsets: naturally occurring thymus-derived Tregs (tTregs) and those conditionally expanded in the periphery from Tcons (pTregs) (10, 14). Additionally, Tregs can be separated into functional subsets according to their surface phenotype. These include a central Treg population, which has circulatory features similar to those of naive Tcons $\left(\mathrm{CCR} 7^{+} \mathrm{CD} 62 \mathrm{~L}^{+}\right)$, and several activated Treg populations that are similar to effector Tcons $\left(\mathrm{CCR} 7{ }^{\text {lo }} \mathrm{CD} 62 \mathrm{~L}^{\text {lo }} \mathrm{CD} 44^{\mathrm{hi}} \mathrm{KLRG} 1^{+} \mathrm{CD} 103^{+}\right.$) (15). Tregs can also be described based upon effector cytokine expression and transcriptomics $(16,17)$. 
Activated Tregs are CD62 $\mathrm{L}^{\mathrm{lo}}$, display signs of recent TCR stimulation, and reside in nonlymphoid tissues including skin, liver, and gut (18), which are also major aGVHD target organs.

Recent studies have described high stimulation-2 (ST2) expression on specialized Tregs and show that they are enriched in nonlymphoid tissues (19-22). ST2, also called IL-33 receptor, has 2 main splice isoforms from a single transcript dependent on the promoter being used: the distal promoter region when bound to GATA transcription factors transcribes the membrane receptor of ST2; and when PMA-responsive elements bind to the proximal promoter, soluble ST2 (sST2) is transcribed and acts as a decoy receptor for IL-33 (23). In the colon, IL-33 signaling through ST2 in Tregs contributes to the maintenance of Foxp3 expression under inflammatory conditions, allowing Tregs to exert their suppressive function in the inflammatory niche by inhibiting the intestinal Teff response (19). ST2 is also required for the development of visceral adipose tissue-resident Tregs, which limits obesity-associated inflammation (21). Recently, a genome-wide DNA methylation landscape showed that ST2 ${ }^{+}$Tregs localize in specialized tissues, specifically in the skin, gonadal visceral adipose tissue, and liver (22). We previously showed that elevated plasma sST2 in mice and humans is a risk factor of therapy-resistant aGVHD and death $(20,24)$. Intestinal stromal, endothelial, and type $1 \mathrm{~T}$ cells were the major cellular source of sST2 during aGVHD (20). This enhanced sST2 presence has been shown to inhibit the production of the type 2 cytokines IL- 4 and IL-5 but not the type 1 cytokine IFN- $\gamma$ (25). We have also shown that ST2 blockade with a neutralizing antibody reduces plasma sST2 as well as frequencies of sST2-producing T cells while maintaining membrane ST2-expressing T cells (Th2 and ST2 ${ }^{+}$Tregs) during aGVHD (20). IL-33 signaling through ST2 helps induce Th2-associated cytokines (26) and IL-9-producing T cells (27), and peri-transplant IL-33 administration allows expansion of Tregs in aGVHD models (28). These findings suggest a nonredundant role of ST2 in functional specialization and Treg homeostasis. Herein, we studied nonlymphoid tissue ST2+ Tregs. We uncovered mechanisms underlying the inverse relationship between ST2 and ROR $\gamma$ t expression in intestinal Tregs during aGVHD, and our work suggests novel therapeutic targets within Tregs during aGVHD and the potential to ultimately establish a practical Tregbased cellular therapy for aGVHD prevention.

\section{Results}

$S T 2^{+}$Tregs are expanded from thymic Tregs and localized in the target organs after HCT. Given their natural presence, high stability, and crucial function in maintaining homeostasis, we hypothesized that ST2 ${ }^{+}$Tregs in nonlymphoid tissues are predominantly thymus derived. To determine whether ST2 ${ }^{+}$Tregs were either thymic (tTregs) or peripheral (pTregs), donor grafts comprising CD25-depleted $\mathrm{T}$ cells and Tregs were sorted from 2 different C57BL/6 (B6) Foxp3 reporter mouse strains to trace the thymic or peripheral origin of donor Tregs, as previously described (29). Mice received WT B6 Foxp3 ${ }^{\text {RFP }}$ CD25-depleted total T cells, and WT or ST2 ${ }^{-/-}$B6 Foxp3 ${ }^{\mathrm{GFP}}$ sorted Tregs with WT B6 T cell-depleted (TCD) BM cells in a minor-mismatched (B6, H-2 $\rightarrow$ C3H.SW, H-2 $2^{\text {b }}$ miH) HCT model. Ex vivo analysis of the gut on day 10 after alloHCT showed a low frequency of Foxp $3^{\text {RFP }}$ in both WT and ST2 ${ }^{-/-}$groups, suggesting minimum de novo generation of pTregs during aGVHD, as has been shown previously (30) (Supplemental Figure 1A; supplemental material available online with this article; https://doi.org/10.1172/jci.insight.122014DS1). In contrast, we found a higher frequency of tTregs in the gut in mice receiving WT Foxp ${ }^{\mathrm{GFP}}$ Tregs compared with those that received ST2 ${ }^{-/-}$Foxp3 ${ }^{\text {GFP }}$ Tregs. Therefore, most Tregs found in the gut were derived from donor tTregs and not from conversion of Tcons to pTregs. Moreover, loss of ST2 on donor tTregs reduced tTreg frequency after allo-HCT in the inflamed aGVHD target organs (gut, liver) but not in secondary lymphoid organs (Supplemental Figure 1A), confirming that ST2 ${ }^{+}$Tregs are tissue-resident Tregs $(21,22)$.

ST2 deficiency on tTregs alters their activation and function after HCT. The finding that mice transplanted with ST2 $2^{-/}$Tregs had a lower frequency of total Foxp3 ${ }^{+}$Tregs (20) suggested that the ST2/IL-33 axis may contribute to activation and function of donor tTregs. In the miH GVHD model with Treg co-adoptive transfer, we found that ST2 ${ }^{-/-}$Foxp3 $3^{\mathrm{GFP}}$ tTregs showed lower expression of the activation markers CD69 and KLRG1, in contrast to WT Foxp3 ${ }^{\mathrm{GFP}}$ tTregs (Supplemental Figure 1B), and gut-localized ST2 ${ }^{-/-}$Foxp3 $3^{\mathrm{GFP}}$ tTregs produced less IL-10 and LAG3 (Supplemental Figure 1C). Of note, no difference in activation markers and IL-10 production was seen for pTregs (data not shown). This is the first analysis to our knowledge of gut-localized donor WT versus ST2 $2^{--}$Tregs that were adoptively transferred before the onset of aGVHD intestinal pathology. The data show that intestinal ST2 ${ }^{+}$Tregs have high KLRG1, CD69, and LAG3 expression, and produce high levels of IL-10. 
Transcriptome analysis of intestinal ST2-1- versus WT Tregs in 2 aGVHD models reveals inverse expression of Illrl1 and Rorc. We have previously shown that co-adoptive transfer of ST2 ${ }^{-1-}$ Foxp3 ${ }^{\text {GFP }}$ Tregs and Tcons eradicates the protective impact of WT Tregs in aGVHD (20). To further understand the mechanism, we performed transcriptome analysis of sorted intestinal Foxp3 ${ }^{\mathrm{GFP}}$ Tregs from aGVHD mice receiving WT CD25-depleted total T cells with either WT or ST2 ${ }^{-/-}$Foxp3 ${ }^{\mathrm{GFP}}$ Tregs in both major-mismatched (B6, $\mathrm{H}-2^{\mathrm{b}} \rightarrow \mathrm{BALB} / \mathrm{c}, \mathrm{H}-2^{\mathrm{d}}$; Mhc) and $\mathrm{miH}$ aGVHD models. We found that ST2 ${ }^{-/-}$versus WT Foxp3 ${ }^{\mathrm{GFP}}$ Tregs showed decreased expression of key molecules needed for Treg function such as Foxp3, Il2ra, Icos, Il27, Ctla4, and Ahr (Figure 1 and Supplemental Table 1). Loss of Foxp3 on Tregs led to increased expression of inflammatory molecules in intestinal ST2 ${ }^{-/-}$versus WT Tregs in both models (Supplemental Table 2). Strikingly, among all the upregulated genes in intestinal ST2 ${ }^{-/-}$Foxp3 ${ }^{\mathrm{GFP}}$ Tregs, transcription of RAR-related orphan receptor $\mathrm{c}$ (Rorc), which encodes ROR $\gamma$ t, the essential gene for proinflammatory IL-17 $7^{+} \mathrm{T}$ cell development $(20,31-33)$, was the most upregulated transcript in ST2 ${ }^{-/-}$versus WT Foxp $3^{\mathrm{GFP}}$ Tregs isolated from the gut in both models (Figure 1). Therefore, we next explored the role of the IL-17 transcription factor Rorc in intestinal Tregs analyzed under steady-state and inflamed gut conditions.

Rorc deficiency shows an increase in ST2 ${ }^{+}$Tregs in the steady-state gut. The transcriptome finding led us to investigate the frequency and function of Tregs in the gut of naive mice deficient for Rorc. Isolation and staining of intestinal $\mathrm{T}$ cells showed a significant increase in Treg frequency in the gut of Rorc ${ }^{-/-}$mice compared with WT mice (Figure 2A). Interestingly, $\sim 55 \%$ of Tregs from Rorc $^{-1-}$ mice were ST2 ${ }^{+}$compared with only $\sim 12 \% \mathrm{ST} 2^{+}$Tregs from WT mice (Figure $2 \mathrm{~B}$ ). Our data suggest that at steady state, Rorc deficiency in intestinal $\mathrm{T}$ cells leads to increased frequency of Foxp $3^{+}$Tregs among all $\mathrm{T}$ cells, particularly $\mathrm{ST}^{+}$Tregs. Rorc $^{-/-}$versus WT mice showed increased total Treg frequencies in liver $(2.74 \% \pm 0.03 \%$ vs. $11.4 \% \pm 0.9 \%$, no difference in ST2 $2^{+}$Tregs), and spleen $(13.8 \% \pm 0.2 \%$ vs. $23.8 \% \pm 1.3 \%$, no difference in $\mathrm{ST}^{+}$Tregs) but not in peripheral blood; lymph nodes were not tested. These data suggest a preferentially nonlymphoid-specific mechanism to support gut ST2 ${ }^{+}$Treg accumulation likely dependent on responses to local endogenous antigen stimulation following HCT, analogous to the local suppressive effects of tTregs in draining lymph nodes of mice with autoimmune ovarian disease (34).

Rorc deficiency alleviates aGVHD severity via increased frequencies of functional ST2 ${ }^{+}$Tregs in the inflamed gut and restoring the Tcon/Treg balance. Mice receiving donor Rorc $^{-/-} \mathrm{T}$ cells showed less-severe aGVHD and increased survival compared with those receiving WT T cells in the miH GVHD model (Figure 2C). Lower aGVHD scores were correlated with decreased systemic SST2 and IFN- $\gamma$ levels in plasma of mice receiving Rorc $^{-1-} \mathrm{T}$ cells (Figure 2D). Infiltrated intestinal Tregs, particularly ST2 ${ }^{+}$Treg frequencies of recipients receiving Rorc $^{-1-} \mathrm{T}$ cells, were significantly higher as compared with those receiving WT $\mathrm{T}$ cells (Figure 2, E and F). Ex vivo analysis of these sorted infiltrated intestinal Tregs from mice receiving Rorc $^{-1-} \mathrm{T}$ cells showed that they expressed significantly higher levels of membrane-bound ST2 than those receiving WT T cells. As expected, sST2 was not detected in Tregs (Figure 2G). These ST2 ${ }^{+}$Tregs derived from Rorc $^{-/-} \mathrm{T}$ cells also showed higher expression of TIGIT and Helios (Figure 2H), suggesting an increased suppressor function compared with WT Tregs even in the context of inflammation, consistent with studies of Treg-specific TIGIT (35) or Helios (36) deletion. We have previously shown that co-adoptive transfer of ST2 ${ }^{+}$Tregs mediated less-severe aGVHD by restoring a Tcon/Treg balance specifically by reducing Tcons producing sST2 and increasing Tcons expressing membrane-bound ST2 (20). Therefore, we next explored whether this counterbalance would also happen with donor Rorc ${ }^{-1-} \mathrm{T}$ cells in mice undergoing HCT. Indeed, we found that Tcons from Rorc $^{-1-}$ recipients produced less sST2 as compared with WT Tcons while expressing higher levels of membrane-bound ST2 than their WT counterparts (Figure 2, I and J). Recipients of Rorc ${ }^{-/-}$donor T cells showed lower frequencies of IFN- $\gamma-$ producing $\mathrm{T}$ cells in the gut on day 14 after HCT (Figure $2 \mathrm{~K}$ ). At the same time, and along with the increased frequency of ST2 ${ }^{+}$Tregs, recipients of $\mathrm{Rorc}^{-/}$donor T cells showed increased percentages of Th2 cytokine IL- 4 and of IL-10 in Tcons (Figure 2L). These data indicate that T cells from Rorc ${ }^{-/-}$mice have a preferential role in exerting a protective effect against aGVHD.

Rorc $^{-1-} S T 2^{+}$Tregs decrease GVHD severity and mortality, increase intestinal ST2 ${ }^{+}$KLRG1 $1^{+}$Tregs, and decrease intestinal type $1 \mathrm{~T}$ cells after HCT through an intrinsic mechanism. To verify whether ST2 ${ }^{+}$Tregs from Rorc ${ }^{-1}$ mice were qualitatively different from WT ST2 ${ }^{+}$Tregs, we sorted ST2 ${ }^{+}$Tregs from Rorc ${ }^{-1}$ or WT mice and cotransplanted each at a 1:10 ratio with CD25-depleted WT T cells in an miH model. ST2 ${ }^{+}$Tregs from Rorc $^{-/-}$mice cotransplanted at the same ratio as WT ST2 ${ }^{+}$Tregs were able to further decrease GVHD scores and mortality (Figure 3A). Consequently, on day 14 after HCT, in recipients receiving Rorc $^{-/-} \mathrm{ST}^{+}$ 

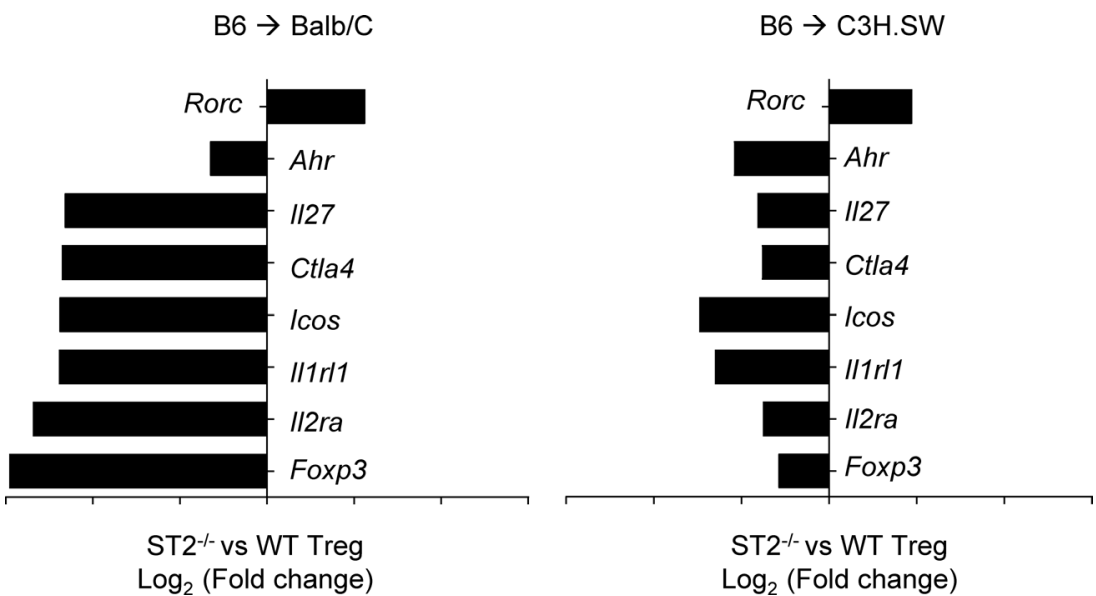

Figure 1. Transcriptome analysis of intestinal ST2 ${ }^{-1-}$ versus WT Tregs in $\mathbf{2}$ aGVHD models. Transcriptome analysis of Foxp3 ${ }^{\text {CFP }}$ Tregs sorted from the gut of a pool of 3-5 BALB/C or C3H.SW mice receiving WT CD25-depleted total T cells with either WT or ST2 ${ }^{-1-}$ Foxp3 $3^{\text {GFP }}$ Tregs (ratio 3:1 for BALB/C and 5:1 for C3H.SW) on day 14 and day 10 after HCT, respectively. Data shown as ratio of fold change between sorted $\mathrm{ST}^{-1-}$ and WT Foxp3 ${ }^{\mathrm{CFP}}$ Tregs $\log _{2}$ transformed.

Tregs, approximately $45 \%$ of intestinal Tregs were ST2 ${ }^{+}$versus $30 \%$ in recipients receiving WT ST2 ${ }^{+}$Tregs (Figure 3B). These Rorc $^{-1-}$ ST2 ${ }^{+}$Tregs after HCT express more KLRG1 and Helios (Figure 3C). We also looked at the impact on effector intestinal $\mathrm{CD} 4^{+}$and $\mathrm{CD} 8^{+} \mathrm{T}$ cells at the same time after HCT. We showed decreased total lymphocyte infiltration (Figure 3D) and lower frequencies of IFN- $\gamma$-producing $\mathrm{CD}^{+}{ }^{+}$and $\mathrm{CD}^{+} \mathrm{T}$ cells in the gastrointestinal tract of mice receiving $\mathrm{Rorc}^{-/-} \mathrm{ST}^{+}$Tregs versus WT ST2 $2^{+}$Tregs (Figure $3 \mathrm{E}$ ). These data suggest an intrinsic role of Rorc in ST2 $2^{+}$Tregs in aGVHD.

Ex vivo polyclonal Tregs cultured with supplemental IL-33 (Treg $\left.{ }_{I L-33}\right)$ express more ST2, activation markers, amphiregulin, and immunosuppressive function compared with those cultured either without IL-33 or with IL-33 and IL-23/IL-17. We next wondered whether we could use these ST2 ${ }^{+}$Tregs as a cellular therapy avenue for preventing GVHD. IL-33 signaling in Tregs has been shown to (i) elicit amphiregulin production that enhances repair ability for injured muscles (37), independent of their suppressive activity on Teffs (38); and (ii) induce superior Teff immunosuppression ex vivo $(19,39)$ and in a colitis model (19). Therefore, we hypothesized that IL-33/ST2 stimulation will drive superior ST2 ${ }^{+}$Treg function through these dual properties. To observe whether IL-33/ST2 upregulation of amphiregulin expression would be advantageous over current Treg culture methods, we used a short-term culture (3 days) of Tregs to facilitate clinical translation. Because IL-23, a key proinflammatory cytokine in the maintenance and amplification of Th17/Tc17, can restrain Treg responses by inhibiting IL-33 responsiveness (19), we tested whether adding IL-23 + IL-17 would subvert the beneficial effect of IL-33. Using ex vivo polyclonal Tregs from B6 donors cultured with IL-2 (control Tregs) versus IL-2 + IL-33 ( Treg $_{\text {IL-33 }}$ ) versus IL-2 + IL-33 + IL-2 $3+$ IL-17, we found that adding IL-33 doubled the frequencies of ST2 ${ }^{+}$Tregs compared with control Tregs, and adding IL-23 + IL-17 reversed this effect (Figure 4A). Markers of Treg activation (KLRG1)

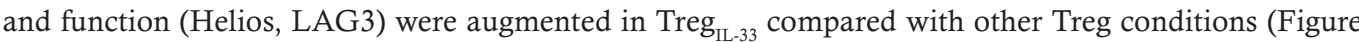
4B). Treg ${ }_{\text {IL-33 }}$ also expressed more amphiregulin protein and RNA than Tregs cultured in either of the 2 other conditions (Figure 4C). To evaluate immunosuppressive function, Tregs were added to $\mathrm{CD} 4^{+}$ and $C D 8^{+}$Teffs at 1:1 to $1: 12$ ratios. The percentage of CD4 suppression by Treg $_{\text {IL-33 }}$ as compared with control Tregs was significant only at a physiological ratio of 1:12; CD8 suppression at a ratio of 1:3 was the most significant (Supplemental Figure 2A). For both CD4 and CD8 proliferation suppression, adding IL-23/IL-17 significantly inhibited the superior immunosuppressive effect of IL-33 starting at ratio of 1:3 for $\mathrm{CD} 4^{+}$Teffs and 1:1 for $\mathrm{CD} 8^{+}$Teffs. To verify whether the enhanced suppressive ability of Treg was directly due to the expression of ST2, WT versus ST2 ${ }^{-/}$Tregs were cultured with/without IL-33 and cocultured with Teffs at Treg/Teff ratios of 1:12 and 1:24 respectively. ST2 $2^{-/-}$Tregs inhibited Teff proliferation to the same extent as WT Tregs in vitro. Treg ${ }_{\mathrm{IL}-33}$ showed enhanced suppressor function on CD4 ${ }^{+}$ $\mathrm{T}$ cells compared with Tregs cultured without IL-33 or ST2 $2^{-/-}$Tregs. No difference was found between $\mathrm{ST}^{-/-}$Tregs cultured with/without IL-33 (Supplemental Figure 2B). These data suggest that Treg $_{\text {IL-33 }}$ 

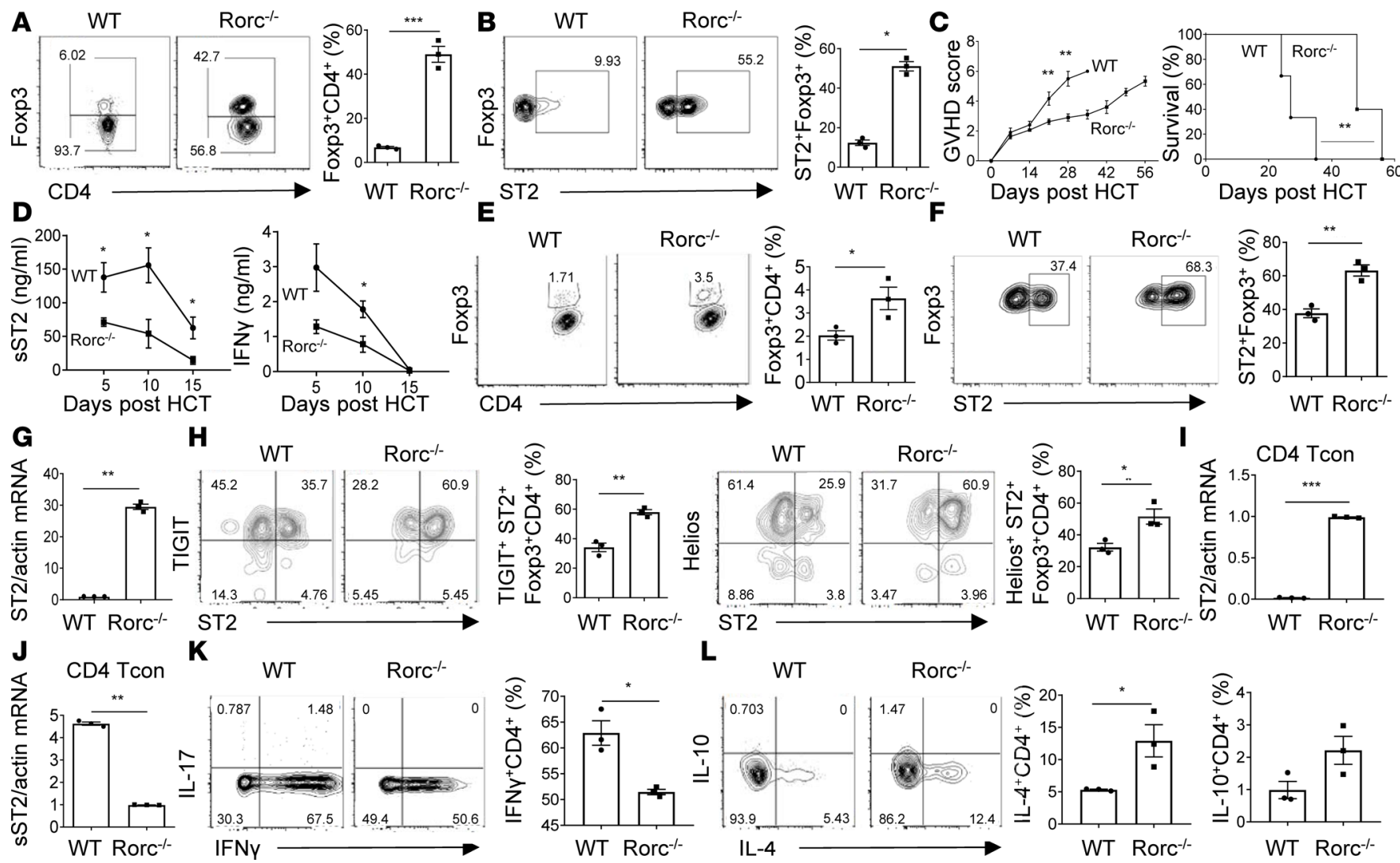

IFNY
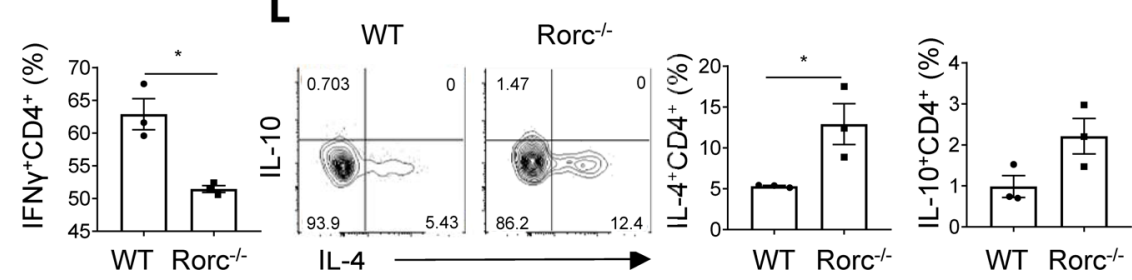

Figure 2. Rorc deficiency alleviates aCVHD severity associated with increased frequencies of functional ST2+ ${ }^{+}$Tregs in the inflamed intestine and restoration of Tcon/Treg balance. (A) Representative plots of Foxp3 expression on CD4 ${ }^{+}$T cells in gut of naive B6 WT or Rorc ${ }^{-1-}$ mice. $n=3$, data are shown as mean $\pm \mathrm{SEM}$; unpaired $t$ test, ${ }^{* *} P<0.001$. (B) Representative plots of ST2 expression on Tregs in gut of naive B6 WT or Rorc ${ }^{-1-}$ mice, respectively. $n=3$, data are shown as mean $\pm \mathrm{SEM}$; unpaired $t$ test, ${ }^{*} P<0.05$. (C) Clinical score and survival curve of lethally irradiated C3H.SW mice receiving B6 WT TCD BM with WT or Rorc ${ }^{-1-}$ donor T cells. $n=5$, data are shown as mean \pm SEM; unpaired $t$ test, ${ }^{* *} P<0.01$ for clinical score; a logrank test was used for survival analysis, ${ }^{* *} P<0.01$. (D) $s S T 2$ and IFN- $\gamma$ concentrations in plasma collected from the mice described above at the indicated time points after HCT. $n=3$, data are shown as mean \pm SEM; unpaired $t$ test, ${ }^{*} P<0.05$. (E) Representative plots of Foxp3 expression on CD4 ${ }^{+}$ T cells isolated from the gut of $\mathrm{C} 3 \mathrm{H}$.SW mice receiving WT or Rorc ${ }^{-1-}$ T cells on day 14 after allo-HCT, $n=3$, data are shown as mean \pm SEM; unpaired $t$ test, ${ }^{*} P<0.05$. (F) Representative plots of ST2 expression on Tregs isolated from the gut of C3H.SW mice receiving WT or Rorc ${ }^{-1-} \mathrm{T}$ cells on day 14 after allo-HCT. $n=3$, data are shown as mean \pm SEM; unpaired $t$ test, ${ }^{* *} P<0.01$. (G) mRNA expression of ST2 on Tregs sorted from the gut of mice in $\mathbf{C}$ on day 14 after allo-HCT. $n=3$, data are shown as mean $\pm \mathrm{SEM}$; unpaired $t$ test, ${ }^{* *} P<0.01$. (H) Representative plots of ST2, TIGIT, and Helios expression on Foxp3 $3^{+}$Tregs from the gut of $\mathrm{C3H}$.SW mice receiving WT or Rorc ${ }^{-1-}$ T cells on day 14 after HCT. $n=3$, data are shown as mean \pm SEM; unpaired $t$ test, ${ }^{*} P<0.05$, ${ }^{* *} P<0.01$. (I and $\mathbf{J}$ ) mRNA expression of ST2 and $\mathrm{sST} 2$ by sorted Foxp $3^{\mathrm{CFP}-}$ effector $\mathrm{T}$ cells isolated from the gut of mice in C on day 14 after HCT. $n=3$, data are shown as mean $\pm \mathrm{SEM}$; unpaired $t$ test, ${ }^{* *} P<0.01$, ${ }^{* * *} P<0.001$. (K and $\mathbf{L}$ ) Representative plots of IFN- $\gamma$, IL-17, IL-4, and IL-10 production by Foxp $3^{\text {CFP- }}$ effector T cells from the gut of C3H.SW mice receiving WT or Rorc ${ }^{-1-}$ T cells on day 14 after HCT. $n=3$, data are shown as mean $\pm \mathrm{SEM}$; unpaired $t$ test, ${ }^{*} P<0.05$.

increase their immunosuppressive capacity on Teffs, while adding Th17-related cytokines to Treg $_{\text {IL-33 }}$ counteracted this effect, as previously shown $(19,39)$.

Adoptive transfer of ex vivo cultured donor Tre ${ }_{\text {LL-33 }}$ leads to superior protection from aGVHD compared with those cultured either without IL-33 or with IL-33 and IL-23/IL-17, and amphiregulin blockade abolishes the protective effect of Treg ${ }_{I L-33}$. We next examined the in vivo capacity of donor Treg ${ }_{\text {IL-33 }}$ to protect from aGVHD intestinal damage by co-adoptive transfer of Tregs cultured in the 3 aforementioned conditions in both Mhc and $\mathrm{miH}$ aGVHD models. We used a more stringent ratio of 1 donor Tregs per 20 donor Tcons (5\% of total T cells). In both aGVHD models, mice receiving Treg ${ }_{\text {IL-33 }}$ at a 1:20 ratio with CD25-depleted total $\mathrm{T}$ cells showed significantly less-severe aGVHD and better survival compared with those receiving Tregs cultured with IL-2 alone or IL-2 + IL-33 + IL-23 + IL-17 (Figure 5A and Supplemental Figure 3A). Ex vivo analysis of intestinal $\mathrm{T}$ cells showed that mice receiving Treg $_{\text {IL-33 }}$ exhibited decreased intestinal lymphocyte infiltration and increased Treg and ST2 ${ }^{+}$Treg frequencies on day 10 after HCT, whereas T-bet-, IFN- $\gamma-$, ROR $\gamma \mathrm{t}-$, and IL-17-expressing Tcons were decreased 
A
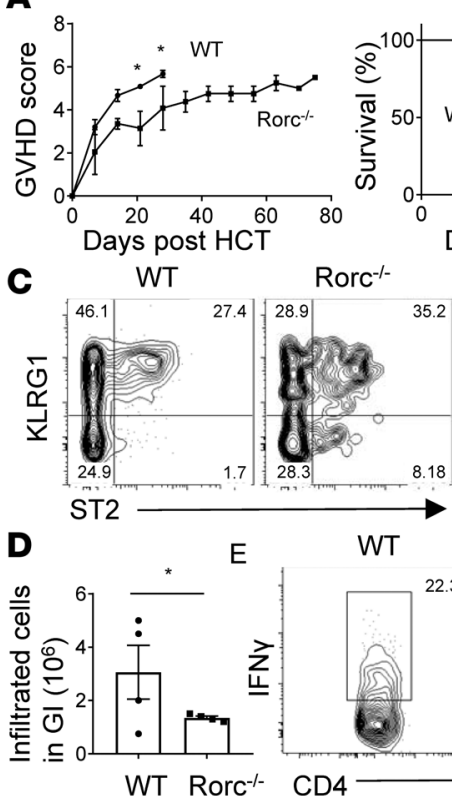

E $\quad$ WT

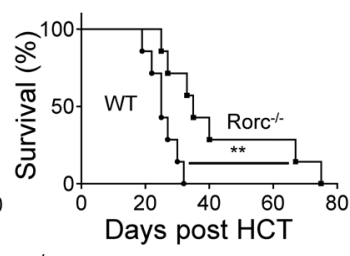

ces
B
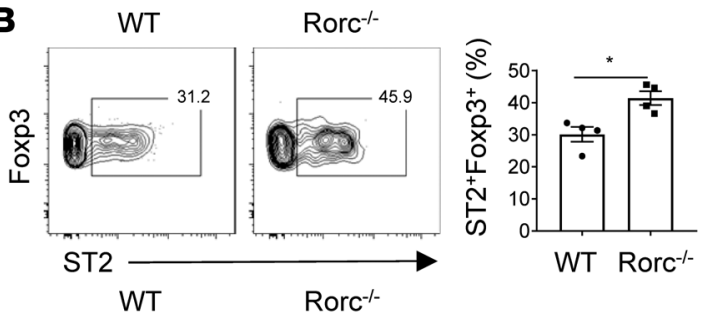

WT Rorc-l-
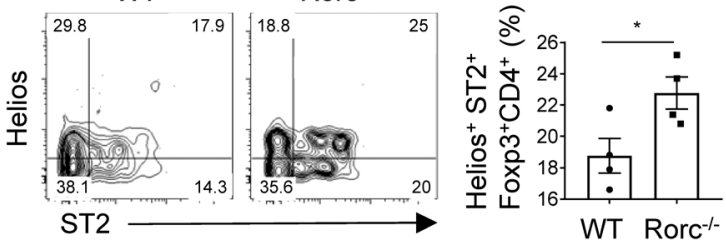
Rorc $^{-1-}$
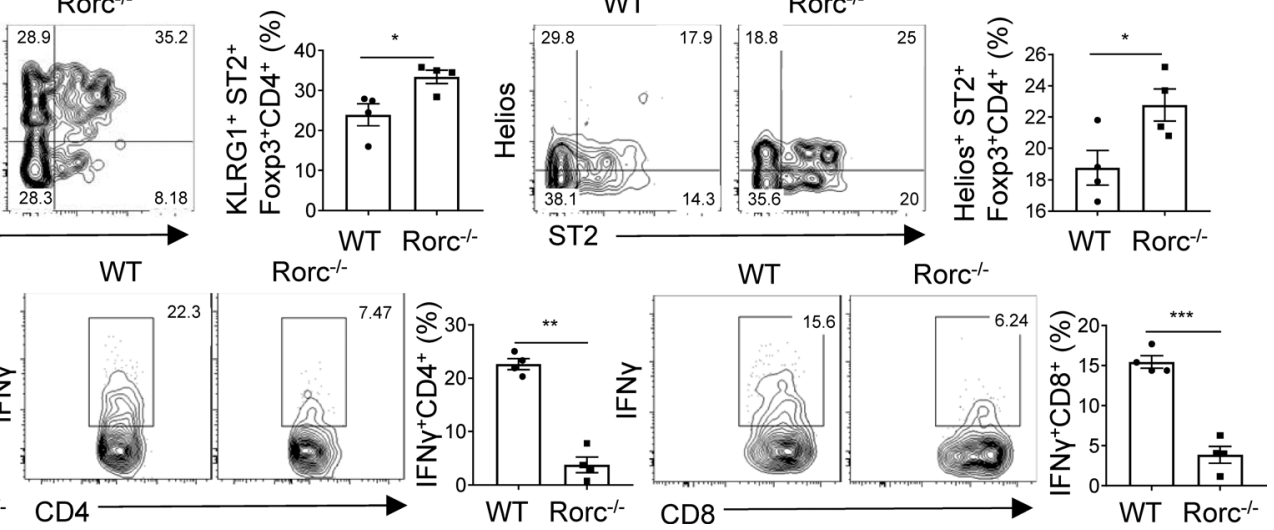

Figure 3. Rorc ${ }^{-1-}$ ST2 ${ }^{+}$Tregs decrease GVHD severity and mortality, increase intestinal ST2 ${ }^{+}$KLRG1+ ${ }^{+}$Tregs after HCT, and decrease intestinal type 1 T cells. (A) Clinical scores and the survival curve of lethally irradiated C3H.SW mice that received B6 WT TCD BM and WT CD25-depleted total T cells with $10 \% \mathrm{ST2}^{+}$Tregs isolated from WT or Rorc ${ }^{-1-}$ mice. $n=7$, from 2 independent experiments, data are shown as mean \pm SEM; unpaired $t$ test, ${ }^{*} P<0.05$ for clinical scores; a log-rank test was used for survival analysis, ${ }^{* *} P<0.01$. (B) Representative plots of ST2 expression on Tregs isolated from the gut of C3H. SW mice in $\mathbf{A}$ receiving WT or Rorc ${ }^{-1-}$ ST2 ${ }^{+}$Tregs analyzed on day 14 after allo-HCT. $n=4$, data are shown as mean \pm SEM; unpaired $t$ test, ${ }^{*} P<0.05$. (C) Representative plots of ST2, KLRG1, and Helios expression on Foxp $3^{+}$Tregs from the gut of C3H.SW mice in A receiving WT or Rorc ${ }^{-1-}$ ST2 ${ }^{+}$Tregs on day 14 after HCT. $n=4$, data are shown as mean \pm SEM; unpaired $t$ test, ${ }^{*} P<0.05$. (D) Total infiltrated cells in the gastrointestinal (CI) tract of mice in $\mathbf{A}$ receiving WT or Rorc ${ }^{-1-}$ ST2 ${ }^{+}$Tregs analyzed on day 14 after HCT. $n=4$, data are shown as mean \pm SEM; unpaired $t$ test, ${ }^{*} P<0.05$. (E) Representative plots of IFN- $\gamma$ production by $\mathrm{CD}^{+}$and $\mathrm{CD} 8^{+}$Teffs from the gut of $\mathrm{C} 3 \mathrm{H}$.SW mice in A receiving WT or Rorc ${ }^{-/-}$ST2 $2^{+}$Tregs analyzed on day 14 after allo-HCT. $n=4$, data are shown as mean $\pm \mathrm{SEM}$; unpaired $t$ test, ${ }^{* *} P<0.01,{ }^{* *} P<0.001$.

and less proliferated (Figure 5B and Supplemental Figure 3B). To verify that the effects of IL-33 on Tregs were also through increased upregulation of amphiregulin, mice cotransferred with Treg $_{\text {IL-33 }}$ were injected with anti-amphiregulin antibody or isotype. We found that amphiregulin blockade in HCT recipients receiving $\operatorname{Treg}_{\mathrm{IL}-33}$ abolished the protective effect of Treg $\mathrm{IL}-33_{3}$ compared with the isotype control (Figure 5C). These data indicated that $\mathrm{Treg}_{\mathrm{IL}-33}$ prevents GVHD through a dual mechanism: immunosuppression of Teffs and upregulation of amphiregulin in $\operatorname{Treg}_{\text {IL33 }}$.

\section{Discussion}

Since we have shown before that ST2 ${ }^{+}$Tregs prevent the development of aGVHD in an experimental model (20), we aimed to investigate the mechanism by which this occurs. Our data first showed that $\mathrm{ST}^{+}$Tregs were expanded from donor tTregs following allo-HCT. They were predominantly localized in aGVHD target organs (gut and liver), but not in the secondary lymphoid organs, as reported for naive mice (22). We also found that absence of ST2 on donor tTregs decreased their activation (CD69, KLRG1) and function (LAG3, IL-10) in the inflamed gut. We then performed transcriptome analysis of intestinal ST2 ${ }^{-/-}$versus WT Tregs from the disparate Mhc and miH GVHD models and showed downregulation of transcripts necessary for the function of Tregs (Foxp3, Ctla4, Il27, Icos, Illrl1, Il2ra, Ahr). As the loss of Foxp3 expression has been shown to be associated with reversion to a more inflammatory phenotype in both transplant and nontransplant models (40-44), we looked at the gene transcription upregulated in intestinal ST2 ${ }^{-/-}$Tregs as compared with intestinal WT Tregs from both HCT models. Most of these genes were indeed involved in the inflammatory profile (Supplemental Table 2). Among these, Rorc was the only upregulated gene in intestinal ST2 $2^{-/-}$Tregs in both HCT models. Tregs can be activated upon induction of peripheral antigen encounter at barrier surfaces (45), which may explain why Rorc was more upregulated in Mhc versus miH GVHD recipients, as target antigen load and strength of the TCR response to Mhc that drives proliferation are typically higher $(46,47)$. 

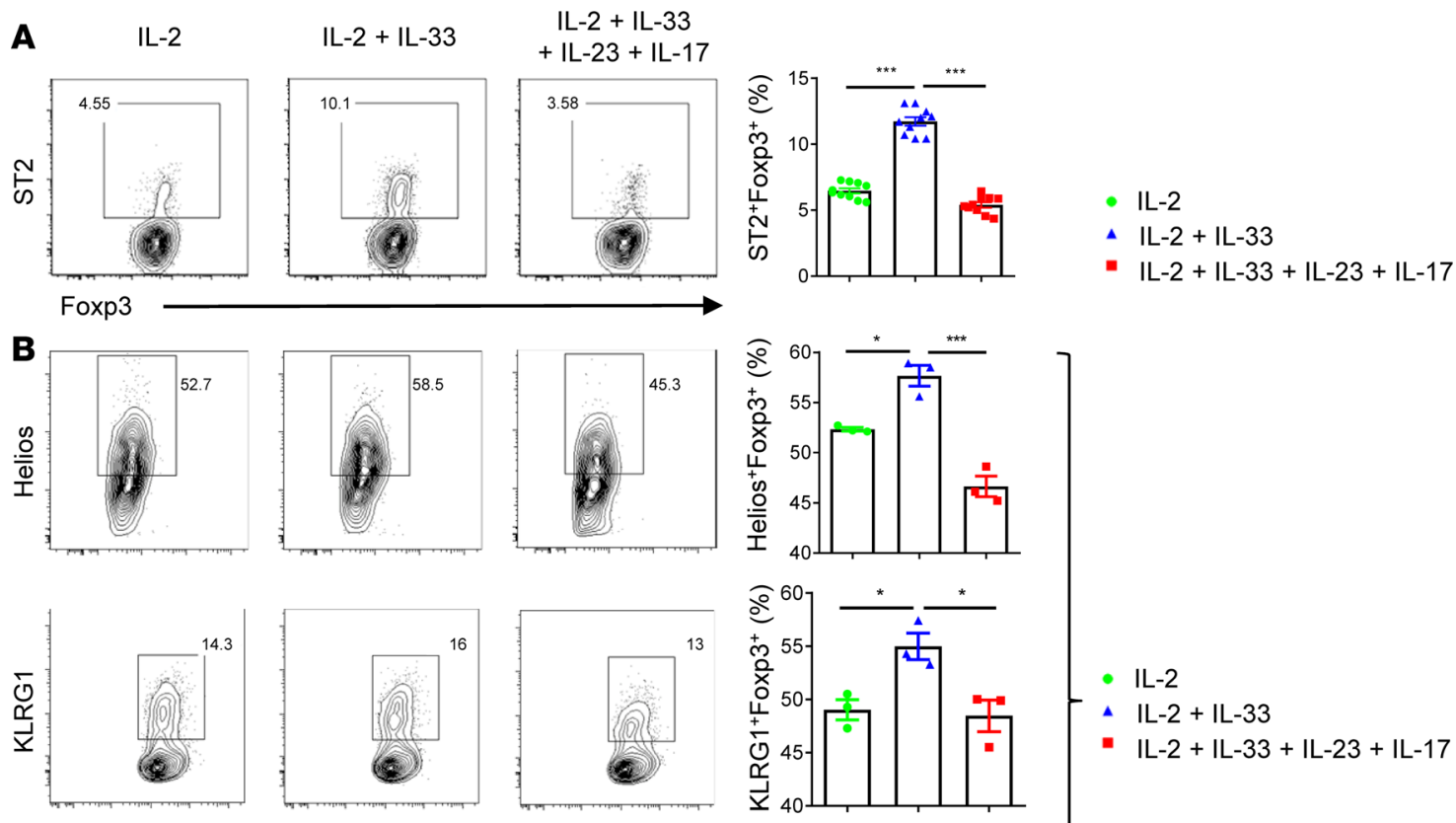

- IL-2

IL-2 + IL-33 + IL-23 + IL-17
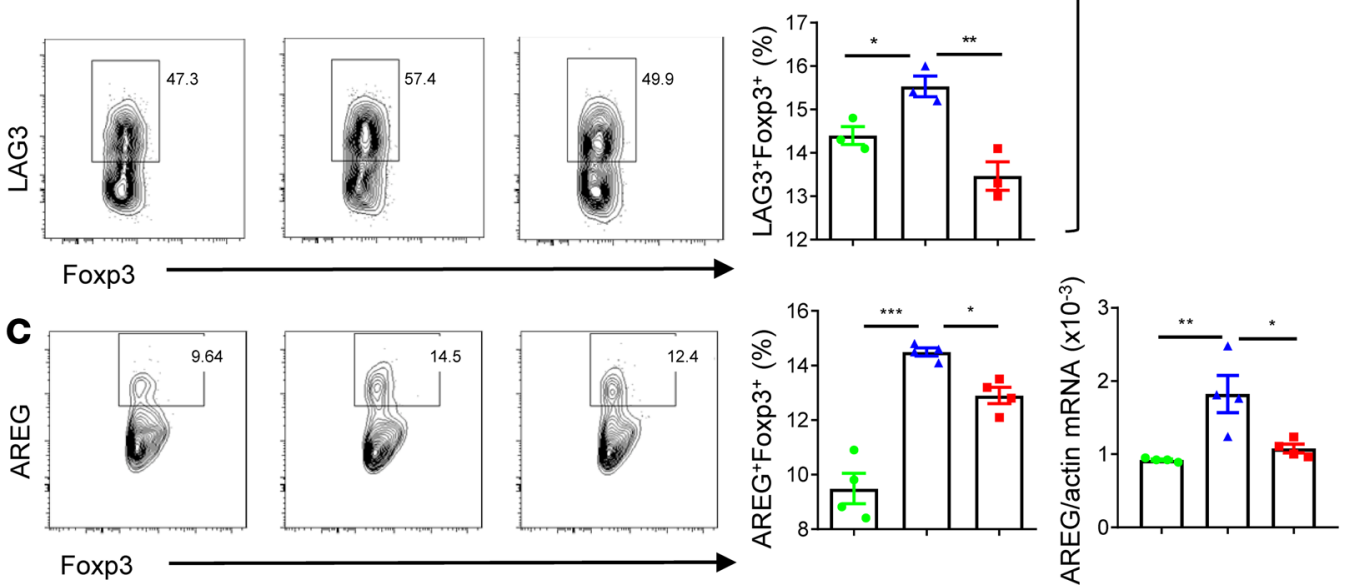

- IL-2

- IL-2 + IL-33

Foxp3

- IL-2 + IL-33 + IL-23 + IL-17

Figure 4. Ex vivo polyclonal Tregs cultured with supplemental IL-33 $\left(\operatorname{Treg}_{\mathrm{IL-33}}\right)$ express more ST2 and activation markers, and amphiregulin as compared with those cultured without IL-33 or inhibited with IL-23/IL-17. (A) Representative plots of ST2 expression on B6 Tregs after different stimulation conditions (IL-2 [2 ng/ml]; IL-2 [2 ng/ml] + IL-33 [20 ng/ml]; IL-2 [2 ng/ml] + IL-33 [20 ng/ml] + IL-17 [40 ng/ml] + IL-23 [40 ng/ml]) for 3 days. Graphs show the frequency of ST2 expression on B6 Tregs. $n=10$, data are shown as mean \pm SEM; ANOVA with Bonferroni's correction for multiple comparisons, ${ }^{* * *} P$ $<0.001$. (B) Representative plots of KLRG1, LAG3, and Helios expression on B6 Tregs after different stimulation conditions (IL-2 [2 ng/ml]; IL-2 [2 ng/mI] + IL-33 [20 ng/ml]; IL-2 [2 ng/ml] + IL-33 [20 ng/ml] + IL-23 [40 ng/ml] + IL-17 [40 ng/ml] ) for 3 days. Graphs show the frequency of expression of KLRG1, LAC3, and Helios on B6 Tregs. $n=3$, from 2 independent experiments, data are shown as mean \pm SEM; ANOVA with Bonferroni correction for multiple comparisons, ${ }^{*} P<0.05,{ }^{* *} P<0.01,{ }^{* *} P<0.001$. (C) Representative plots of amphiregulin (AREC) expression on B6 Tregs after different stimulation conditions (IL-2 [2 ng/ml]; IL-2 [2 ng/ml] + IL-33 [20 ng/ml]; IL-2 [2 ng/ml] + IL-33 [20 ng/ml] + IL-23 [40 ng/ml] + IL-17 [40 ng/ml]) for 3 days. For AREG analysis, cells were stimulated for 3 hours with PMA and ionomycin in the presence of monensin. Graphs show the frequency and mRNA expression of AREG on B6 Tregs. $n=4$, data are shown as mean \pm SEM; ANOVA with Bonferroni's correction for multiple comparisons, ${ }^{*} P<0.05,{ }^{* *} P<0.01$, ${ }^{* * *} P<0.001$.

Inflammatory signals, particularly cytokines, augment the differentiation of activated $\mathrm{T}$ cells from resting $\mathrm{T}$ cells that circulate through the blood and lymphatics to reside in tissues rather than secondary lymphoid organs (18). Disease suppression by Tregs depends on endogenous antigen stimulation at the location in which Tregs reside that leads to the accumulation of antigen-specific Tregs that can continuously negate pathogenic $\mathrm{T}$ cell response (48). Therefore, we next explored the role of the IL-17 transcription factor Rorc in intestinal Tregs analyzed under steady state and inflamed gut conditions. In steady state, we showed that Tregs from Rorc $^{-/-}$mice were more frequent and expressed higher levels of ST2 compared with those from WT mice. Knocking down Rorc on T cells showed a reduction in GVHD severity (49), in accordance with our observation; here we showed that Tregs from $\mathrm{Rorc}^{-/-}$mice were more frequent and expressed higher levels of membrane-bound ST2, TIGIT, and Helios than those from WT mice during intestinal inflammation. 
A

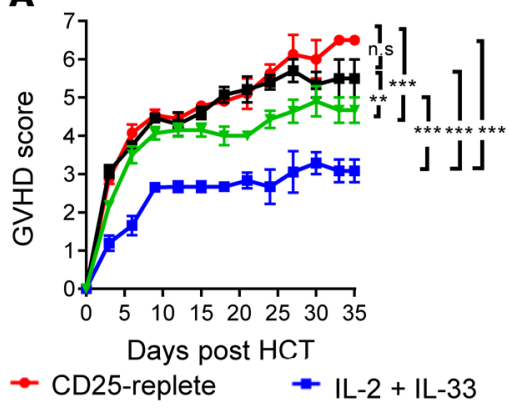

$=\mathrm{IL}-2=\mathrm{IL}-2+\mathrm{IL}-33+\mathrm{IL}-23+\mathrm{IL}-17$
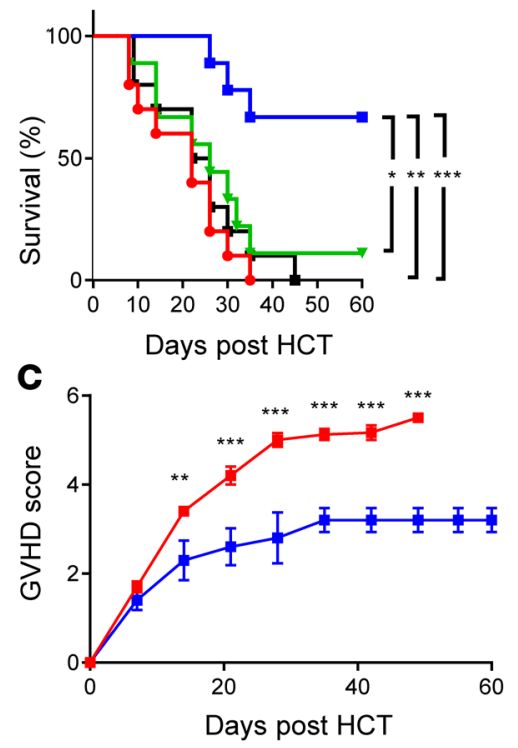

B
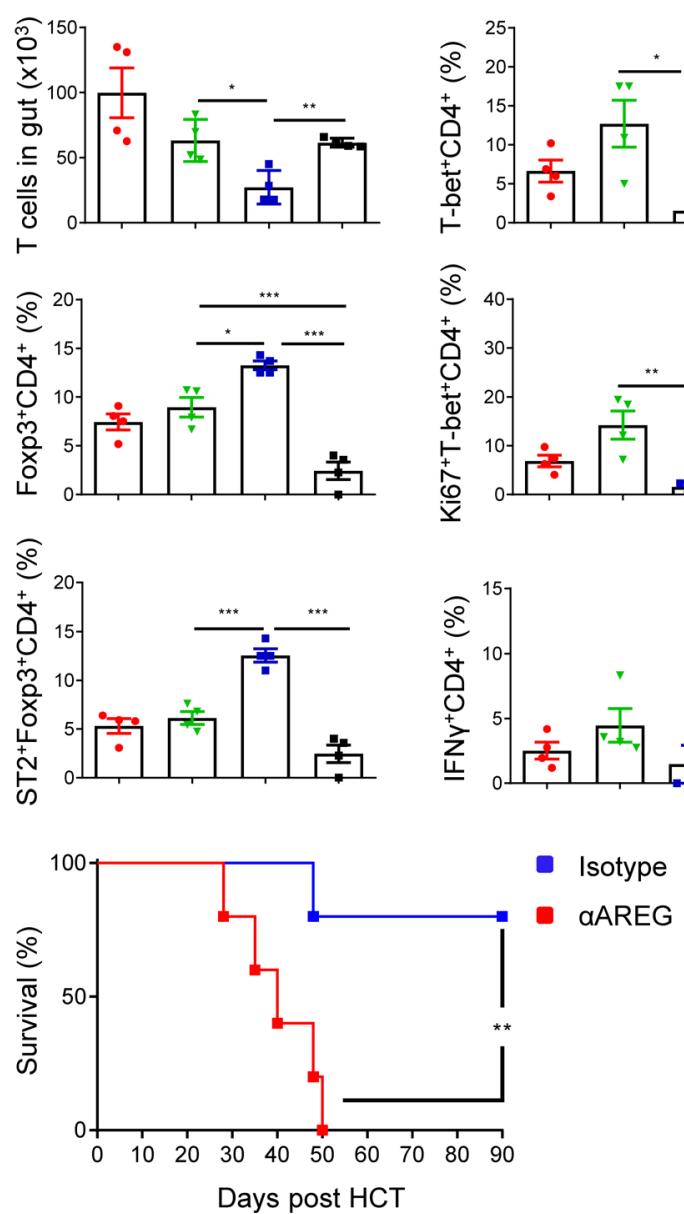
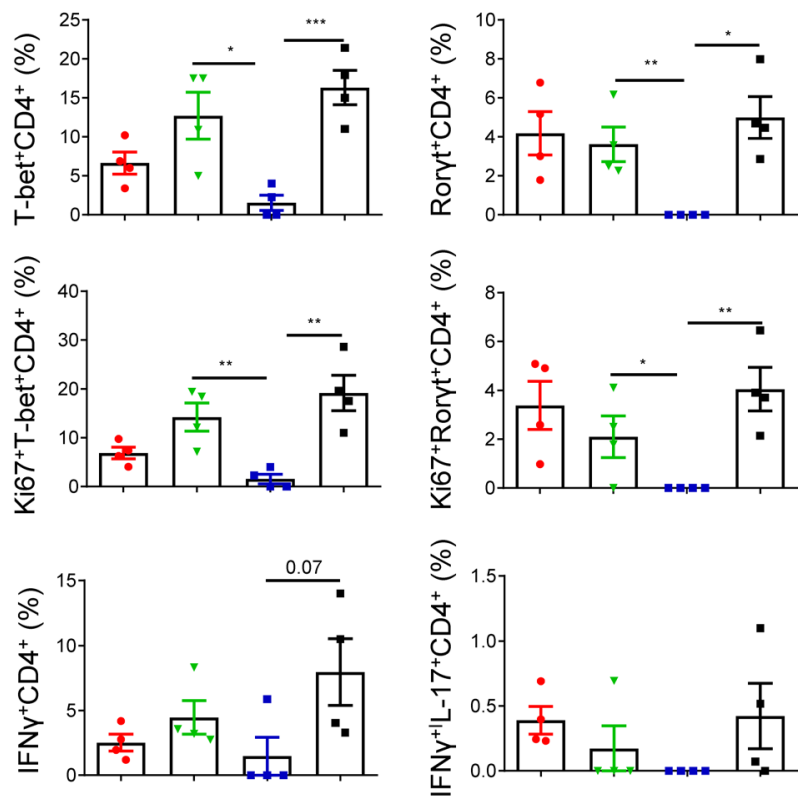

Figure 5. Donor Treg ${ }_{\mathrm{IL}-33}$ improve GVHD compared with Tregs cultured without IL-33 or with IL-33 + IL-23/17, and AREC blockade abolishes the protective effect of Treg ${ }_{1 \mathrm{~L}-33^{*}}$ (A) Clinical score of aCVHD and survival curve for BALB/c mice receiving allogenic B6 CD25-replete T cells or CD25-depleted total T cells with ex vivo cultured Tregs (IL-2; IL-2 + IL-33; IL-2 + IL-33 + IL-23 + IL-17), ratio of 20:1. $n=10$ per group, data are shown as mean \pm SEM; ANOVA with Bonferroni's correction for multiple comparisons, ${ }^{* *} P<0.01,{ }^{* * *} P<0.001$ for clinical score; a log-rank test was used for survival analysis, ${ }^{*} P<0.05,{ }^{* *} P<0.01,{ }^{* * *} P<$ 0.001. (B) Graphs represent total infiltrated cells in gut, and frequency of Foxp3, T-bet, ROR $\gamma \mathrm{t}$, Ki-67, ST2, IFN- $\gamma$, IL-17 in infiltrated T cells isolated from the gut of BALB/c recipient mice on day 10 after allo-HCT. $n=4$, data are shown as mean \pm SEM; ANOVA with Bonferroni's correction for multiple comparisons, ${ }^{*} P<0.05$, ${ }^{* *} P<0.01,{ }^{* * *} P<0.001$. (C) Clinical score of aCVHD and survival curve for C3H.SW mice receiving allogenic B6 CD25-depleted total T cells with ex vivo cultured Treg ${ }_{\mid--33}$, ratio of 10:1. Mice were treated with five 100- $\mu$ g doses of anti-amphiregulin or isotype control every other day from day -1 to day 7. $n=5$ per group, data are shown as mean $\pm \mathrm{SEM}$; unpaired $t$ test, ${ }^{* *} P<0.001$ for clinical score; a log-rank test was used for survival analysis, ${ }^{* *} P<0.01$.

This led to a shift in the balance of Tcons, with decreased type 1 cells that secreted less SST2 and expressed more ST2 on their surface compared with WT T cells. In the present study, several mechanisms are possible: (i) cell-intrinsic, similar to a study showing the E3 ubiquitin ligase Itch is a negative regulator of IL-17A production in colonic inflammation through targeting of the transcription factor ROR $\gamma t$ for proteasomal degradation (50); and (ii) indirect, favored by the intestinal cytokine and antigen milieu $(18,48,51)$. Transplantation with Rorc $^{-1-} \mathrm{T}$ cells has previously been shown to greatly diminish aGVHD severity (49, 52-56), but transplantation with Rorc ${ }^{-/}$Tregs, or ST2 $2^{+}$Tregs has not to our knowledge been done before. Since Tregs, particularly ST2 ${ }^{+}$Tregs, were enriched in Rorc $^{-/-}$mice, it was important to verify whether ST2 ${ }^{+}$ Tregs in Rorc $^{-/-}$mice were qualitatively different from those in WT mice. It has been shown that in transplant recipient mice, donor WT naive OT-II ${ }^{+} \mathrm{CD} 4^{+} \mathrm{T}$ cells developed into $\mathrm{ROR} \gamma \mathrm{t}^{+}$Tregs when the mice were fed ovalbumin, whereas when mice received cells deficient in ROR $\gamma$ t, a majority developed into Gata3 ${ }^{+}$ Tregs, showing that a loss in ROR $\gamma t$ affected Tregs through a cell-intrinsic pathway (57). In the current study, we show that ST2 ${ }^{+}$Tregs from Rorc $^{-/}$mice were able to decrease GVHD score and mortality as compared with ST2 ${ }^{+}$Tregs from WT mice, which indicated that ST2 was intrinsically associated with Rorc on Tregs.

We have recently shown in murine and human models that IL-9-producing T cells stimulated by the ST2/IL-33 pathway (T9 ${ }_{\text {IL-33 }}$ ) expressed amphiregulin and provided protection against epithelial damage 
from aGVHD (27). We proposed use of $\mathrm{T}_{\text {IL-33 }}$ in the context of malignant setting when GVL activity is needed. In the current study, we propose the use of Treg ${ }_{\text {IL-33 }}$ as a cellular therapy to prevent GVHD in the context of a nonmalignant setting, as Tregs need not have anticancer activity. Indeed, adoptive transfer of ex vivo cultured donor Tregs has been shown by several groups in experimental (5-7) and clinical aGVHD (8-10). Several methods of activation of Tregs for translational purposes have been approved (58-61), and we propose here a new approach with a potential new mechanism of Treg function. Studies have shown that IL-33 activation of ST2 ${ }^{+}$Tregs increases their suppressive function $(19,20)$. Peri-administration of IL-33 expands recipient Tregs that protect mice against aGVHD (28). The administration of recombinant IL-33 substantially expanded tissue ST2 ${ }^{+}$Tregs in all organs assessed: the number of tissue-resident ST2 ${ }^{+}$Tregs increased by 10-fold in fat, 5-fold in skin, 13-fold in liver, and 60-fold in lung tissue (22). These findings suggested that ST2 ${ }^{+}$Tregs were a distinct state in tissues and underwent population expansion via IL-33. We found that $\operatorname{Treg}_{\mathrm{IL}-33}$ were able to suppress CD4 ${ }^{+}$Teffs only slightly more than control Tregs in vitro at lower Treg/Teff ratios. These data were partly in contrast to the finding that showed suppressive function of IL-33-cultured Tregs was not impaired at higher Treg/Teff ratios (19). This could be due to different experimental conditions as well as different Treg/Teff ratios. Indeed, in the latter study, Tregs and Teffs were cocultured in the presence of IL-33 without prior in vitro activation of Tregs with IL-33. In addition, the Treg/Teff ratio was higher (1:1 and 1:3). In our conditions, we first cultured Tregs with IL-2 + IL-33 for 3 days to induce ST2 expression, then cocultured them with Teffs under different Treg/Teff ratios (up to 1:24). As expected and similar to the above-mentioned study (19), ST2 ${ }^{-/}$Tregs did not show additional suppression ability, as Tregs did not respond to IL-33, indicating the suppression ability of Treg $_{\text {IL-33 }}$ was directly due to the expression of ST2. In line with the previous study, we found adoptive transfer of Treg ${ }_{\mathrm{IL}-33}$ ameliorated aGVHD via inhibiting the Teff response in vivo. To explain the more modest suppressor function in the in vitro compared with the in vivo setting, we hypothesized that the substantial increase in GVHD inhibition in vivo by Treg $_{\text {IL-33 }}$ may be due to increased expression in Treg $_{\text {IL-33 }}$ of amphiregulin that may contribute to GVHD inhibition as a result of its tissue-reparative properties (shown in Figure 5C). Alternatively, the fact that LAG3, Helios, and KLRG1 levels were higher in 3-day in vitro cultures of Tregs containing IL-33 may have contributed to improved GVHD protective effects by Treg $_{\text {IL33 }}$. Indeed, one of the important characteristics that was found for tissue-resident ST2 ${ }^{+}$Tregs in skin and fat was the production of amphiregulin (22), which facilitated repair from muscle damage (37) and viral infection (38). Studies have suggested that IL-33 also promotes amphiregulin expression on ILC2s, leading to intestinal tissue protection dependent on amphiregulin-EGFR interactions (62). Blocking amphiregulin after adoptive transfer of $\mathrm{T} 9_{\mathrm{IL}-33}$ has been shown to reduce allogeneic mouse and human colonic epithelial survival in vitro and increase aGVHD severity in vivo (27). In the current study we showed that $\operatorname{Treg}_{\mathrm{IL}-33}$ also expressed higher amphiregulin in vitro. Importantly, blocking amphiregulin by neutralizing antibody in vivo abolished the protective effect of Treg ${ }_{\mathrm{IL}-33}$ in the miH HCT model. Therefore, adoptive transfer of ex vivo cultured donor Treg ${ }_{\text {IL-33 }}$ better protected from aGVHD intestinal damage compared with Tregs cultured without IL-33, possibly via synergistic immunosuppressive and amphiregulin-EGFR tissue protection mechanisms. Our data confirmed that adoptive transfer of Treg $_{\mathrm{IL}-33}$ is a viable therapeutic option.

Finally, we investigated whether inflammatory IL-23 and IL-17 can interfere with ST2 signaling in Tregs, and showed that IL-23 and IL-17 inhibit the effect of IL-33 on Tregs both ex vivo and in vivo as shown with IL-23 on IL-33-induced pTregs in a colitis model (19). We did not find differences in Rorc expression on Tregs cultured under the 3 aforementioned conditions. It has been shown previously that IL-23 does not induce Rorc or Th17 cytokines in CD4 ${ }^{+} \mathrm{T}$ cells isolated from a collagen-induced arthritis murine model (63). In addition, Schiering et al. showed that IL-23 limited T cell responsiveness to IL-33 by inhibiting expression of GATA3 and ST2 in colonic Tregs in vitro and in vivo (19). We analyzed the whole transcriptome data published by Schiering et al. and compared Rorc gene expression in colonic effector $\mathrm{CD}^{+} \mathrm{T}$ cells between WT and IL-23r ${ }^{-1}$ mice; however, we did not find any difference (data not shown). We hypothesized that the short-term culture of Tregs with IL-33 + IL-23 could not induce Rorc expression. Therefore, for allo-HCT, adding IL-33 to Treg expansion cultures might improve their protective effects in aGVHD. The effect of IL-33 on Tregs could be inhibited by adding IL-23 and IL-17 as in a colitis model (19), suggesting that early administration of $\operatorname{Treg}_{\mathrm{IL}-33}$, before development of an overwhelming Th17/Tc17 response, should be favored.

We conclude that within GVHD target organs, ST2 ${ }^{+}$Tregs are predominantly intestine localized, and inversely correlated with ROR $\gamma t$ in steady-state and inflammatory conditions. Triggering the ST2/ 
IL-33 pathway in Tregs prevents severe aGVHD, possibly through a dual mechanism: upregulation of amphiregulin and immunosuppression on Teffs. Thus, Treg ${ }_{\mathrm{IL}-33}$ represents a potential cellular therapy avenue for preventing aGVHD.

\section{Methods}

Mice. B6 (B6, H-2 b CD45.2 $)$, B6 Foxp3 ${ }^{\mathrm{RFP}}$, B6 Rorc ${ }^{-/}$, BALB/c (H-2 $)$, and C3H.SW (H-2 $\left.{ }^{\mathrm{b}}, \mathrm{CD} 45.2^{+}\right)$ mice were purchased from the Jackson Laboratory. B6 Foxp $3^{\mathrm{GFP}}$ mice were provided by Matthew J. Turner (Indiana University); B6 ST2 ${ }^{-/-}\left(\mathrm{CD} 45.2^{+}\right)$mice were provided by Andrew McKenzie (University of Cambridge, Cambridge, United Kingdom) and were bred to B6 Foxp3 $3^{\mathrm{GFP}}$, and the homozygous ST2 ${ }^{-/-}$Foxp3 ${ }^{\mathrm{GFP}}$ genotype was confirmed by PCR.

Induction and assessment of aGVHD. Mice underwent allo-BM transplantation as previously described $(20,64)$. Briefly, BALB/c and C3H.SW recipients received 900 and 1100 cGy total body irradiation (x-ray or ${ }^{137} \mathrm{Cs}$ source, respectively) on day -1 . Recipient mice were injected intravenously with TCD BM cells (5 $\times 10^{6}$ ) plus splenic T cells, $2 \times 10^{6}$ for C3H.SW or $1 \times 10^{6}$ for BALB/c allogeneic donors, on day 0 . T cells from donor mice were enriched using the murine Pan T Cell Isolation Kit (Miltenyi Biotec), and TCD BM was prepared with CD90.2 Microbeads (Miltenyi Biotec). Mice were housed in sterilized Micro-Isolator cages and maintained on acidified water $(\mathrm{pH}<3)$ for 3 weeks. Survival was monitored daily, and clinical GVHD scores were assessed weekly as described previously (65). Mice were euthanized when clinical scores reached 6.5, in accordance with animal protocols approved by the Institutional Review Board.

Isolation of intestinal cells. Single-cell suspensions were prepared from intestines as described previously (20). Briefly, intestines were flushed with PBS to remove fecal matter and mucus. Fragments $(<0.5 \mathrm{~cm})$ of intestines were digested in $10 \mathrm{ml}$ DMEM containing collagenase type B ( $2 \mathrm{mg} / \mathrm{ml}$; Roche), deoxyribonuclease I (10 $\mu \mathrm{g} / \mathrm{ml}$; Roche), and $4 \%$ bovine serum albumin (Sigma-Aldrich) at $37^{\circ} \mathrm{C}$ with shaking for 90 minutes. The digested mixture was then diluted with $30 \mathrm{ml}$ plain DMEM, filtered through a $70-\mu \mathrm{m}$ strainer and centrifuged at $850 \mathrm{~g}$ for 10 minutes. The cell pellets were suspended in $5 \mathrm{ml}$ of $80 \%$ Percoll (GE Healthcare), overlaid with $8 \mathrm{ml}$ of $40 \%$ Percoll and spun at $1000 \mathrm{~g}$ for 20 minutes at $4^{\circ} \mathrm{C}$ without braking. Enriched lymphocytes were collected from the interface. At the University of Minnesota, after intestines were flushed with PBS to remove mucus and fecal matter, Peyer patches were removed. Digestion of tissue occurred over a 2-step process. Single-cell suspensions from 2 incubations of the tissue fragments $(1-1.5 \mathrm{~cm})$ in $10 \mathrm{ml}$ PBS containing $5 \mathrm{mM}$ EDTA, $10 \mathrm{mM} \mathrm{HEPES}$, and $2 \% \mathrm{FCS}$ at $37^{\circ} \mathrm{C}$ for 10 minutes each were collected through a $70-\mu \mathrm{m}$ strainer. Remaining tissue was cut into smaller fragments $(<0.5 \mathrm{~cm})$ and digested in $10 \mathrm{ml}$ RPMI containing 5\%FCS, $50 \mathrm{U} / \mathrm{ml}$ Dispase (Sigma-Aldrich), $0.5 \mathrm{mg} / \mathrm{ml}$ of each Collagenase D (Roche) and DNase I (Sigma-Aldrich) for 60 minutes at $37^{\circ} \mathrm{C}$, collecting single-cell suspensions through a $70-\mu \mathrm{m}$ strainer and adding fresh digestion solution to the remaining tissue at 20 -minute intervals. The digested tissue was filtered through a $70-\mu \mathrm{m}$ strainer and centrifuged at $2000 \mathrm{~g}$ for 10 minutes. The cell pellets were suspended in $5 \mathrm{ml}$ of $80 \%$ Percoll (GE Healthcare), overlaid with $8 \mathrm{ml}$ of $40 \%$ Percoll, and spun at $1400 \mathrm{~g}$ for 20 minutes at $20^{\circ} \mathrm{C}$ without braking. The enriched lymphocyte layer was collected from the interface.

Flow cytometry. All antibodies and reagents for flow cytometry were purchased from eBioscience, unless stated otherwise (see Supplemental Table 3). The cells were preincubated with purified anti-mouse CD16/ $\mathrm{CD} 32 \mathrm{mAb}$ for $10-20$ minutes at $4^{\circ} \mathrm{C}$ to prevent nonspecific binding of the antibodies. The cells were subsequently incubated for 30 minutes at $4^{\circ} \mathrm{C}$ with antibodies for surface staining. Fixable viability dye (FVD) was used to distinguish live cells from dead cells. The Foxp3/Transcription Factor Staining Buffer Set and the Fixation and Permeabilization Kit (Invitrogen) were used for intracellular transcription factor and cytokine staining. For cytokine staining, cells were restimulated with PMA (50 ng/ml), ionomycin (1 $\mu \mathrm{g} / \mathrm{ml}$; Sigma-Aldrich), and brefeldin A for 4-6 hours before any staining. For the analysis of amphiregulin production, ex vivo cultured Tregs were stimulated with PMA (50 ng/ml) and ionomycin (1 $\mathrm{nM})$ for 3 hours in the presence of GolgiStop (monensin).

Cell sorting. Intestinal Tregs and $\mathrm{CD}^{+}$Tcons from $\mathrm{C} 3 \mathrm{H}$.SW recipient mice receiving allo-HCT were sorted based on Foxp $3^{\mathrm{GFP}}$ expression. Foxp $3^{\mathrm{GFP}}$-expressing cells were collected from aGVHD mice on day 10 or day 14 after transplantation of allogeneic and further characterized by quantitative reverse transcription PCR (qPCR) and NanoString analysis. Cell sorting was performed using a BD FACSAria (BD Bioscience).

$q P C R$. Total RNA was isolated from sorted cells using the RNeasy Plus Mini Kit (QIAGEN). cDNA was prepared with a SuperScript VILO cDNA Synthesis Kit (Invitrogen). qPCR was performed using SYBR Green PCR mix on an ABI Prism 7500HT (Applied Biosystems). Thermocycler conditions included 
2-minute incubation at $50^{\circ} \mathrm{C}$, then $95^{\circ} \mathrm{C}$ for 10 minutes; this was followed by a 2-step PCR program of $95^{\circ} \mathrm{C}$ for 5 seconds and $60^{\circ} \mathrm{C}$ for 60 seconds for 40 cycles. $\beta$-Actin was used as an internal control. The primer sequences were as follows: actin forward 5'-CTCTGGCTCCTAGCACCATGAAGA-3', reverse 5'-GTAAAACGCAGCTCAGTAACAGTCCG-3'; ST2 forward 5'-AAGGCACACCATAAGGCTGA-3', reverse 5'-TCGTAGAGCTTGCCATCGTT-3'; sST2 forward 5'-TCGAAATGAAAGTTCCAGCA-3', reverse 5'-TGTGTGAGGGACACTCCTTAC-3'; amphiregulin forward 5'-GGACAATGCAGGGTAAAAGTTGA-3', reverse 5'-TGAAAGAAGGACCAATGTCATTTC-3'.

NanoString. Sorted Foxp $3^{\mathrm{GFP}+}$ Tregs from aGVHD mice were directly lysed in RLT buffer (QIAGEN) on ice. The cell concentration for lysis was $0.2 \times 10^{4}$ to $1 \times 10^{4}$ cells $/ \mu 1$ in a total volume of $5 \mu 1$ with RLT buffer. Lysis samples were immediately frozen in liquid nitrogen and then stored at $-80^{\circ} \mathrm{C}$ or in dry ice. NanoString analysis was performed with the nCounter Analysis System at NanoString Technologies. The nCounter Mouse Immunology Kit, which includes 561 immunology-related mouse genes, was used in the study.

ELISA. Concentrations of IFN- $\gamma$ in plasma were measured with DuoSet ELISA Kits; sST2 was measured with Quantikine ELISA Kits (R\&D Systems).

Treg isolation and stimulation. Mouse Tregs were isolated magnetically from fresh splenocytes of Foxp3 ${ }^{\mathrm{GFP}}$ B6 mice. Cells were plated at a concentration of $1 \times 10^{6} \mathrm{cells} / \mathrm{ml}$ and activated with $1 \mu \mathrm{g} / \mathrm{ml}$ plate-bound anti-CD3 (2C11) and 5-10 $\mu \mathrm{g} / \mathrm{ml}$ soluble anti-CD28 (clone 37.51). Tregs were under different stimulation conditions: IL-2 (2 ng/ml); IL-2 (2 ng/ml) and IL-33 (20 ng/ml); IL-2 (2 ng/ml), IL-33 (20 ng/ml), IL-23 (40 $\mathrm{ng} / \mathrm{ml})$, and IL-17 (40 $\mathrm{ng} / \mathrm{ml})$ for 3 days.

Immunosuppression assays. The ex vivo suppressive capacity of cultured Tregs was assessed with a CTV inhibition assay as previously published (66). Briefly, $\mathrm{CD} 4^{+}$and $\mathrm{CD} 8^{+}$Teffs and antigen-presenting cells (APCs) were purified from B6 mice splenocytes. Teffs were labeled with CTV (Invitrogen) and plated together with the cultured Tregs at different ratios together with APCs in the presence of anti-CD3 (0.25 $\mu \mathrm{g} / \mathrm{ml})$. After 4 days, proliferation of Teffs was measured by flow cytometry.

Adoptive transfer of $\mathrm{ST2}^{+}$Tregs. For the adoptive transfer model in minor-mismatched mice (B6, $\mathrm{H}-2^{\mathrm{b}} \rightarrow \mathrm{C} 3 \mathrm{H} . \mathrm{SW}, \mathrm{H}-2^{\mathrm{b}}$ ), Rorc $^{-/-}$or WT B6 CD4 ${ }^{+} \mathrm{T}$ cells were isolated from Foxp3 ${ }^{\mathrm{GFP}}$ mice using murine $\mathrm{CD}^{+} \mathrm{CD} 25^{+}$Regulatory $\mathrm{T}$ Cell Isolation Kit (Miltenyi Biotec). Tregs were isolated by using APC antimouse CD25 (eBioscience) and an Anti-APC MultiSort Kit (Miltenyi Biotec). ST2 ${ }^{+}$Tregs were further separated by using PE anti-mouse ST2 (MD bioproduct) and anti-PE microbeads (Miltenyi Biotec). A ratio of $1 \mathrm{ST}^{+}$Treg per $10 \mathrm{CD} 25$-depleted total T cells (10\% of total T cells in the graft) was used. TCD BM cells from WT B6 mice were prepared with CD90.2 Microbeads (Miltenyi Biotec).

Adoptive transfer of ex vivo cultured Treg ${ }_{I L-33}$ and control Tregs. For adoptive transfer models in major-mismatched (B6, H-2 $\rightarrow$ BALB/c, H-2 ${ }^{\mathrm{d}}$ ) and minor-mismatched (B6, H-2 $\rightarrow$ C3H.SW, H-2 ${ }^{b}$ ) mice, first donor B6 Tregs were isolated from Foxp $3^{\mathrm{GFP}}$ mice using murine $\mathrm{CD} 4^{+} \mathrm{CD} 25^{+}$Regulatory $\mathrm{T}$ Cell Isolation Kit (Miltenyi Biotec) and ex vivo cultured for 3 days under the different conditions described above (IL-2, IL-2 + IL-33, IL-2 + IL-33 + IL-17 + IL-23). The day of the transplantation, total B6 donor T cells were purified using the murine Pan T Cell Isolation Kit (Miltenyi Biotec), then CD25-depleted using the murine $\mathrm{CD} 4{ }^{+} \mathrm{CD} 25^{+}$Regulatory $\mathrm{T}$ Cell Isolation Kit (Miltenyi Biotec). A more stringent ratio of 1 cultured Treg per 20 CD25-depleted total T cells (5\% of total T cells in the graft) was used for the 3 cultured Tregs conditions. As a control, one HCT group was transplanted with CD25-replete donor T cells without supplemental Tregs, thus containing a physiological ratio of Tregs. TCD BM cells from donors were prepared with CD90.2 Microbeads (Miltenyi Biotec).

In vivo treatment with anti-AREG. C3H.SW recipients received $1,100 \mathrm{cGy}$ total body irradiation $\left({ }^{137} \mathrm{Cs}\right.$ source) on day -1 . On day 0 , mice were injected intravenously with B6 TCD BM cells $\left(5 \times 10^{6}\right), 2 \times 10^{6}$

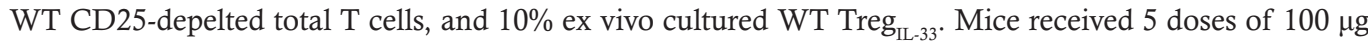
anti-AREG or isotype control every other day from day -1 to day 7 .

Microarray analysis. Microarray data were downloaded in the Gene Expression Omnibus under accession number GSE58164 (19). Microarray was performed using GeneSpring GX12 software (Agilent). Data were normalized using $75 \%$ percentile shift normalization algorithm and baseline transformed to the median of all samples. Statistical significance was determined using an unpaired $t$ test followed by Benjamini-Hochberg FDR multiple testing correction. The $P$ value cutoff was set to 0.05 .

Statistics. Phenotypic and functional data were compared using unpaired $t$ test for 2-group comparison and ANOVA test for comparison of 3 or more group. Prior to analysis, the normality assumption was examined. All tests were 2 sided at the significance level $P<0.05$. To account for the type I error inflation 
due to multiple comparisons, we applied Bonferroni's correction. A log-rank test was used for survival analysis. The analyses were performed using Prism software version 7.03 (GraphPad), and data are presented as mean \pm SEM.

Study approval. Animal experiments and euthanasia protocols performed in this study were approved by the Indiana University School of Medicine IACUC (protocol 18033).

\section{Author contributions}

JY, ML, AR, and DKR designed and performed research, analyzed data, and wrote the manuscript; JZ, $\mathrm{BG}, \mathrm{HL}$, and $\mathrm{KLH}$ performed research and analyzed data; BRB designed experiments, analyzed data, and was involved in data discussions and manuscript editing; SP conceived the project, designed experiments, analyzed data, and wrote the manuscript.

\section{Acknowledgments}

The authors are supported by grants from the National Cancer Institute (R01CA168814, U01CA232491, R01 HL11879) and the Leukemia \& Lymphoma Society (no. 1293-15). We thank Andrew McKenzie for providing the $\mathrm{ST} 2^{-/-}$mice.

Address correspondence to: Sophie Paczesny, Indiana University, Wells Center For Pediatric Research, 1044 W. Walnut Street, Room R4-425, Indianapolis, Indiana 46202, USA. Phone: 317.278.5487; E-mail: sophpacz@iu.edu. Or to: Bruce R. Blazar, University of Minnesota, Department of Pediatrics, Masonic Cancer Center, 420 Delaware Street SE, MMC 109, Minneapolis, Minnesota, 56456, USA. Phone: 612.273.2800; Email: blaza001@umn.edu.

1. Paczesny S, Hanauer D, Sun Y, Reddy P. New perspectives on the biology of acute GVHD. Bone Marrow Transplant. 2010;45(1):1-11.

2. Khera N, Emmert A, Storer BE, Sandmaier BM, Alyea EP, Lee SJ. Costs of allogeneic hematopoietic cell transplantation using reduced intensity conditioning regimens. Oncologist. 2014;19(6):639-644.

3. Zeiser R, Blazar BR. Acute graft-versus-host disease — biologic process, prevention, and therapy. NEngl J Med. 2017;377(22):2167-2179.

4. Jones SC, Murphy GF, Korngold R. Post-hematopoietic cell transplantation control of graft-versus-host disease by donor CD425 T cells to allow an effective graft-versus-leukemia response. Biol Blood Marrow Transplant. 2003;9(4):243-256.

5. Hoffmann P, Ermann J, Edinger M, Fathman CG, Strober S. Donor-type CD4(+)CD25(+) regulatory T cells suppress lethal acute graft-versus-host disease after allogeneic bone marrow transplantation. J Exp Med. 2002;196(3):389-399.

6. Edinger M, et al. CD4+CD25+ regulatory T cells preserve graft-versus-tumor activity while inhibiting graft-versus-host disease after bone marrow transplantation. Nat Med. 2003;9(9):1144-1150.

7. Taylor PA, Lees CJ, Blazar BR. The infusion of ex vivo activated and expanded CD4(+)CD25(+) immune regulatory cells inhibits graft-versus-host disease lethality. Blood. 2002;99(10):3493-3499.

8. Martelli MF, et al. HLA-haploidentical transplantation with regulatory and conventional T-cell adoptive immunotherapy prevents acute leukemia relapse. Blood. 2014;124(4):638-644.

9. Brunstein CG, et al. Infusion of ex vivo expanded $\mathrm{T}$ regulatory cells in adults transplanted with umbilical cord blood: safety profile and detection kinetics. Blood. 2011;117(3):1061-1070.

10. Di Ianni M, et al. Tregs prevent GVHD and promote immune reconstitution in HLA-haploidentical transplantation. Blood. 2011;117(14):3921-3928.

11. Liston A, Gray DH. Homeostatic control of regulatory T cell diversity. Nat Rev Immunol. 2014;14(3):154-165.

12. Taylor PA, et al. L-Selectin(hi) but not the L-selectin(lo) CD4+25+ T-regulatory cells are potent inhibitors of GVHD and BM graft rejection. Blood. 2004;104(12):3804-3812.

13. Ermann J, et al. Only the CD62L+ subpopulation of CD4+CD25+ regulatory T cells protects from lethal acute GVHD. Blood. 2005;105(5):2220-2226.

14. Ohkura N, Kitagawa Y, Sakaguchi S. Development and maintenance of regulatory T cells. Immunity. 2013;38(3):414-423

15. Campbell DJ, Koch MA. Phenotypical and functional specialization of FOXP3+ regulatory T cells. Nat Rev Immunol. 2011;11(2):119-130

16. Cretney E, Kallies A, Nutt SL. Differentiation and function of Foxp3(+) effector regulatory T cells. Trends Immunol. 2013;34(2):74-80

17. Duhen T, Duhen R, Lanzavecchia A, Sallusto F, Campbell DJ. Functionally distinct subsets of human FOXP3+ Treg cells that phenotypically mirror effector Th cells. Blood. 2012;119(19):4430-4440.

18. Li MO, Rudensky AY. T cell receptor signalling in the control of regulatory T cell differentiation and function. Nat Rev Immunol. 2016;16(4):220-233

19. Schiering C, et al. The alarmin IL-33 promotes regulatory T-cell function in the intestine. Nature. 2014;513(7519):564-568.

20. Zhang J, et al. ST2 blockade reduces sST2-producing T cells while maintaining protective mST2-expressing T cells during graftversus-host disease. Sci Transl Med. 2015;7(308):308ra160. 
21. Vasanthakumar A, et al. The transcriptional regulators IRF4, BATF and IL-33 orchestrate development and maintenance of adipose tissue-resident regulatory T cells. Nat Immunol. 2015;16(3):276-285.

22. Delacher M, et al. Genome-wide DNA-methylation landscape defines specialization of regulatory T cells in tissues [published corrigendum in Nat Immunol. 2017;18(12):1361]. Nat Immunol. 2017;18(10):1160-1172.

23. Griesenauer B, Paczesny S. The ST2/IL-33 axis in immune cells during inflammatory diseases. Front Immunol. 2017;8:475.

24. Vander Lugt MT, et al. ST2 as a marker for risk of therapy-resistant graft-versus-host disease and death. N Engl J Med. 2013;369(6):529-539.

25. Oshikawa K, Yanagisawa K, Tominaga S, Sugiyama Y. Expression and function of the ST2 gene in a murine model of allergic airway inflammation. Clin Exp Allergy. 2002;32(10):1520-1526.

26. Schmitz J, et al. IL-33, an interleukin-1-like cytokine that signals via the IL-1 receptor-related protein ST2 and induces T helper type 2-associated cytokines. Immunity. 2005;23(5):479-490.

27. Ramadan A, et al. Specifically differentiated T cell subset promotes tumor immunity over fatal immunity. J Exp Med. 2017;214(12):3577-3596.

28. Matta BM, et al. Peri-alloHCT IL-33 administration expands recipient T-regulatory cells that protect mice against acute GVHD. Blood. 2016;128(3):427-439

29. Ganguly S, et al. Donor CD4+ Foxp3+ regulatory T cells are necessary for posttransplantation cyclophosphamide-mediated protection against GVHD in mice. Blood. 2014;124(13):2131-2141.

30. Bucher C, et al. IL-21 blockade reduces graft-versus-host disease mortality by supporting inducible T regulatory cell generation. Blood. 2009;114(26):5375-5384.

31. Ivanov II, et al. The orphan nuclear receptor RORgammat directs the differentiation program of proinflammatory IL-17+ T helper cells. Cell. 2006;126(6):1121-1133.

32. Li W, et al. Proteomics analysis reveals a Th17-prone cell population in presymptomatic graft-versus-host disease. JCI Insight. 2016;1(6):e92111.

33. Furlan SN, et al. Systems analysis uncovers inflammatory Th/Tc17-driven modules during acute GVHD in monkey and human T cells. Blood. 2016;128(21):2568-2579.

34. Suissa S, Kezouh A, Ernst P. Inhaled corticosteroids and the risks of diabetes onset and progression. Am J Med. 2010;123(11):1001-1006.

35. Kurtulus S, et al. TIGIT predominantly regulates the immune response via regulatory T cells. J Clin Invest. 2015;125(11):4053-4062.

36. Sebastian M, Lopez-Ocasio M, Metidji A, Rieder SA, Shevach EM, Thornton AM. Helios controls a limited subset of regulatory T cell functions. J Immunol. 2016;196(1):144-155.

37. Burzyn D, et al. A special population of regulatory T cells potentiates muscle repair. Cell. 2013;155(6):1282-1295.

38. Arpaia N, et al. A distinct function of regulatory T cells in tissue protection. Cell. 2015;162(5):1078-1089.

39. Turnquist HR, et al. IL-33 expands suppressive CD11b+ Gr-1(int) and regulatory T cells, including ST2L+ Foxp3+ cells, and mediates regulatory T cell-dependent promotion of cardiac allograft survival. J Immunol. 2011;187(9):4598-4610.

40. Beres A, Komorowski R, Mihara M, Drobyski WR. Instability of Foxp3 expression limits the ability of induced regulatory T cells to mitigate graft versus host disease. Clin Cancer Res. 2011;17(12):3969-3983.

41. Chen X, et al. Blockade of interleukin- 6 signaling augments regulatory T-cell reconstitution and attenuates the severity of graftversus-host disease. Blood. 2009;114(4):891-900.

42. Williams LM, Rudensky AY. Maintenance of the Foxp3-dependent developmental program in mature regulatory T cells requires continued expression of Foxp3. Nat Immunol. 2007;8(3):277-284.

43. Zhou X, et al. Instability of the transcription factor Foxp3 leads to the generation of pathogenic memory T cells in vivo. Nat Immunol. 2009;10(9):1000-1007.

44. Bailey-Bucktrout SL, et al. Self-antigen-driven activation induces instability of regulatory T cells during an inflammatory autoimmune response. Immunity. 2013;39(5):949-962.

45. Rosenblum MD, Gratz IK, Paw JS, Lee K, Marshak-Rothstein A, Abbas AK. Response to self antigen imprints regulatory memory in tissues. Nature. 2011;480(7378):538-542.

46. Suchin EJ, Langmuir PB, Palmer E, Sayegh MH, Wells AD, Turka LA. Quantifying the frequency of alloreactive T cells in vivo: new answers to an old question. J Immunol. 2001;166(2):973-981.

47. Kaplan DH, Anderson BE, McNiff JM, Jain D, Shlomchik MJ, Shlomchik WD. Target antigens determine graft-versus-host disease phenotype. J Immunol. 2004;173(9):5467-5475.

48. Samy ET, Parker LA, Sharp CP, Tung KS. Continuous control of autoimmune disease by antigen-dependent polyclonal CD4+CD25+ regulatory T cells in the regional lymph node. J Exp Med. 2005;202(6):771-781.

49. Fulton LM, et al. Attenuation of acute graft-versus-host disease in the absence of the transcription factor ROR $\gamma \mathrm{t}$. J Immunol. 2012;189(4):1765-1772.

50. Kathania M, et al. Itch inhibits IL-17-mediated colon inflammation and tumorigenesis by ROR- $\gamma$ t ubiquitination. Nat Immunol. 2016;17(8):997-1004.

51. Smigiel KS, et al. CCR7 provides localized access to IL-2 and defines homeostatically distinct regulatory T cell subsets. $J$ Exp Med. 2014;211(1):121-136.

52. Carlson MJ, West ML, Coghill JM, Panoskaltsis-Mortari A, Blazar BR, Serody JS. In vitro-differentiated TH17 cells mediate lethal acute graft-versus-host disease with severe cutaneous and pulmonary pathologic manifestations. Blood. 2009;113(6):1365-1374.

53. Kappel LW, et al. IL-17 contributes to CD4-mediated graft-versus-host disease. Blood. 2009;113(4):945-952.

54. Yi T, et al. Absence of donor Th17 leads to augmented Th1 differentiation and exacerbated acute graft-versus-host disease. Blood. 2008;112(5):2101-2110.

55. Gong H, et al. IL-17C Mitigates murine acute graft-vs.-host disease by promoting intestinal barrier functions and treg differentiation. Front Immunol. 2018;9:2724.

56. Varelias A, et al. Acute graft-versus-host disease is regulated by an IL-17-sensitive microbiome. Blood. 2017;129(15):2172-2185.

57. Ohnmacht C, et al. Mucosal immunology. The microbiota regulates type 2 immunity through ROR $\gamma \mathrm{t}(+) \mathrm{T}$ cells. Science. 2015;349(6251):989-993. 
58. Schreiber TH, et al. Therapeutic Treg expansion in mice by TNFRSF25 prevents allergic lung inflammation. J Clin Invest. 2010;120(10):3629-3640.

59. Wolf D, et al. Superior immune reconstitution using Treg-expanded donor cells versus PTCy treatment in preclinical HSCT models. JCI Insight. 2018;3(20):e121717.

60. Wolf $\mathrm{D}$, et al. Marked in vivo donor regulatory T cell expansion via interleukin-2 and TL1A-Ig stimulation ameliorates graftversus-host disease but preserves graft-versus-leukemia in recipients after hematopoietic stem cell transplantation. Biol Blood Marrow Transplant. 2017;23(5):757-766.

61. Gedaly R, et al. mTOR Inhibitor everolimus in regulatory t cell expansion for clinical application in transplantation [published online head of print, November 16, 2018]. Transplantation. https://insights.ovid.com/crossref?an=00007890-900000000-96296.

62. Monticelli LA, Osborne LC, Noti M, Tran SV, Zaiss DM, Artis D. IL-33 promotes an innate immune pathway of intestinal tissue protection dependent on amphiregulin-EGFR interactions. Proc Natl Acad Sci USA. 2015;112(34):10762-10767.

63. Mus AM, et al. Interleukin-23 promotes Th17 differentiation by inhibiting T-bet and FoxP3 and is required for elevation of interleukin-22, but not interleukin-21, in autoimmune experimental arthritis. Arthritis Rheum. 2010;62(4):1043-1050

64. Aoyama K, et al. Inhibiting retinoic acid signaling ameliorates graft-versus-host disease by modifying T-cell differentiation and intestinal migration. Blood. 2013;122(12):2125-2134.

65. Cooke KR, et al. An experimental model of idiopathic pneumonia syndrome after bone marrow transplantation: I. The roles of minor H antigens and endotoxin. Blood. 1996;88(8):3230-3239.

66. Hippen KL, et al. Massive ex vivo expansion of human natural regulatory T cells (T(regs)) with minimal loss of in vivo functional activity. Sci Transl Med. 2011;3(83):83ra41. 